\title{
Comment on 'The latency period of mesothelioma among a cohort of British asbestos workers
} (1978-2005)': the effect of left censoring

\author{
A Farioli ${ }^{\star}, 1,2,3$, S Mattioli ${ }^{1}$, S Curti ${ }^{1}$ and F S Violante ${ }^{1}$
}

${ }^{1}$ Department of Medical and Surgical Sciences (DIMEC), University of Bologna, Via Palagi 9, 40138 Bologna, Italy; ${ }^{2}$ Department of Environmental Health (Environmental \& Occupational Medicine \& Epidemiology), Harvard School of Public Health, 677 Huntington Ave, Boston, MA 02115, USA and ${ }^{3}$ The Cambridge Health Alliance, Harvard Medical School, 1493 Cambridge St, Cambridge, MA 02139, USA

Sir,

We read with extreme interest the article by Frost on mesothelioma latency period among asbestos workers (Frost, 2013). Despite the main aim of the study being to investigate 'the determinants of mesothelioma latency among a cohort of asbestos workers, paying particular attention to indicators of intensity of asbestos exposure' (Frost, 2013), the reported lack of evidence of an association between duration of exposure and latency attracted some attention. Commenters argued that right censoring occurring in the underlying cohort could have biased an analysis restricted to cases (Consonni et al, 2014; Mirabelli and Zugna, 2014).

We would like to highlight a different pitfall of Frost's analysis. She observed huge differences in median latency periods across categories of year of first exposure to asbestos. Indeed, the median latency for deaths among workers exposed to asbestos before 1940 was 48 years, whereas a median latency of only 11 years characterised cases exposed not earlier than 1980. This difference translated in a dramatic decrease of time ratios with increasing year of first exposure. However, it is surprising that the author interpreted these data without considering the limited follow-up window (1978-2005). On the one hand, all the cases exposed before 1940 entered the analysis with a latency of at least 38 years, as all deaths that occurred before 1978 were left censored. On the other hand, the maximum latency observable for subjects exposed after 1980 was just 25 years as events potentially occurring after 2005 were right censored. To describe how left and right censoring can affect the estimates presented by Frost, we conducted a simple simulation study. We assumed a true latency period constant across categories of the year of first exposure to asbestos based on a gamma distribution (shape parameter 11, scale parameter 3). This distribution has a median of 32 years, in line with the median mesothelioma latency reported for occupational mesothelioma and presented by Frost in the discussion section of her paper (Lanphear and Buncher, 1992). In our simulation, we considered six categories for the year of first exposure (as in Frost's article) and we assumed 300 mesothelioma deaths within each group. For pragmatic reasons, within each category we used the mid-point (i.e. $1935,1945,1955,1965,1975$, and 1985) as the first year of exposure. Figure 1 presents the results from our simulation. On the basis of these assumptions, there is no difference among the categories of first year of exposure when the follow-up is complete and there are no censored cases (Panel A). However, in the presence of left and right censoring, a spurious strong association between time to mesothelioma death and year of first exposure to asbestos is observed (Panel B). This fact is the consequence of the differential loss to follow-up with respect to the exposure categories. In Figure 1, we show the estimates from a single simulation. Nonetheless, we performed a further analysis based on 10000 repetitions and the findings were highly consistent with those presented in this letter (data not shown).

It is remarkable that our estimates are not so far from those reported in Table 2 of Frost's manuscript and this fact suggests that a strong bias might affect her findings. Remarkably, a spurious association between the year of the first exposure and mesothelioma latency might bias all the multivariate time ratios presented in Table 3 . Indeed, all the estimates for variables putatively correlated with the year of first exposure (e.g. main occupation, as the proportion of subjects employed in manufacturing and removal has changed over time) could be biased by the improper adjustment for a covariate spuriously associated with the outcome. Furthermore, left censoring could also directly bias the estimates for other time variables. Compared to the later cohorts, the group of workers exposed for the first time to asbestos before 1950 is likely to have a longer average duration of exposure as well as a spuriously longer average latency. This fact could contribute to explain the

Simulation study of mesothelioma latency after asbestos exposure

Panel A: complete follow-up

$\frac{1}{5}$
응
은
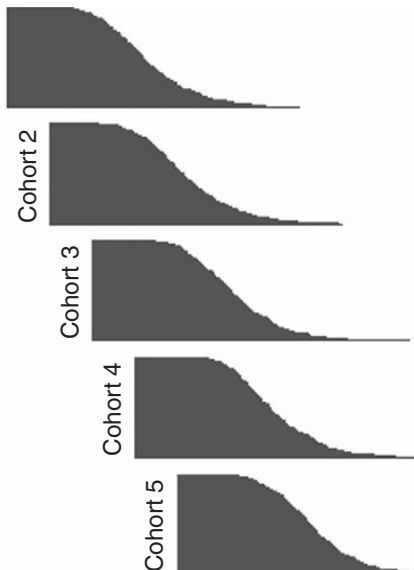

Observed deaths: 300
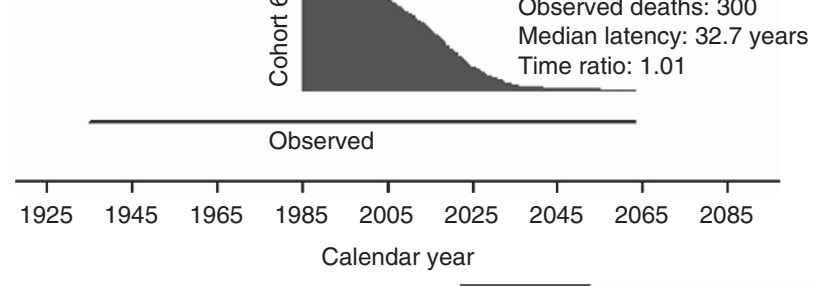

Observed deaths

Panel B: left- and right-censoring

Time ratio: 1.01

-
등
등

Observed deaths: 300

Median latency: 31.2 years

Time ratio: 0.99

Observed deaths: 300 Median latency: 32.3 years

Time ratio: 1.02

Observed deaths: 300

Median latency: 31.5 years

Time ratio: 1.01

Observed deaths: 300

Median latency: 31.2 years

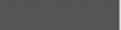

Observed deaths: 300

Median latency: 31.7 years

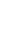


increase in time ratios observed across categories of duration at the univariate analysis (Table 2).

Noteworthy, as the information on asbestosis was available only from 1978, Frost restricted the follow-up window even though information on mesothelioma deaths was available from 1972. This choice determined both a loss of cases and an exacerbation of the bias due to left censoring. Remarkably, asbestosis was only weakly associated with the outcome; hence, this variable is not likely to induce substantial confounding. Thus, Frost should have considered the entire follow-up period to study the other variables. Estimates restricted to 1978 and 2005 and adjusted by asbestosis could have served as a sensitivity analysis.

We would invite Frost to perform a simple and quick reanalysis. She should (i) include only cases exposed for the first time between 1950 and 1969 to limit left and right censoring (she would still retain $56 \%$ of the cases); (ii) analyse the entire follow-up period (1972-2005) to limit left censoring and increase the number of cases; (iii) avoid adjustment for the year of first exposure. If confounding by the latter is a strong concern, Frost could conduct stratum-specific analysis.
The number of deaths that occurred among subjects exposed for the first time between 1950 and 1959 (216 or more after the addition of the years from 1972 to 1977 to the follow-up), and 1960 and 1969 (145 or more) is large enough to fit regression models with a reasonable number of covariates. We believe that this supplemental analysis could add an important piece of knowledge.

\section{REFERENCES}

Consonni D, Barone-Adesi F, Mensi C (2014) Comment on 'The latency period of mesothelioma among a cohort of British asbestos workers (1978-2005)': methodological problems with case-only survival analysis. $\mathrm{Br} J$ Cancer 111: 1674.

Frost G (2013) The latency period of mesothelioma among a cohort of British asbestos workers (1978-2005). Br J Cancer.

Lanphear BP, Buncher CR (1992) Latent period for malignant mesotheliomaof occupational origin. J Occup Med 34: 718-721.

Mirabelli D, Zugna D (2014) Comment on 'The latency period of mesothelioma among a cohort of British asbestos workers (1978-2005)'. Br J Cancer 111: 1675.

*Correspondence: Dr A Farioli; E-mail: andrea_farioli@yahoo.it

Published online 5 August 2014

(c) 2014 Cancer Research UK. All rights reserved 0007-0920/14

\section{(c) (i) (2)(2)}

http://creativecommons.org/licenses/by-nc-sa/3.0/

\section{Response to comment on 'The latency period of mesothelioma among a cohort of British asbestos workers (1978-2005)}

\section{G Frost ${ }^{\star}, 1$}

\section{${ }^{1}$ Mathematical Sciences Unit, Health \& Safety Laboratory, Harpur Hill, Buxton, Derbyshire SK17 9JN, UK}

Sir,

I read with interest the comments by Farioli et al (2014) on the recent publication investigating the latency period of mesothelioma (Frost, 2013). They raised important points regarding the decisions made during analysisin particular, adjusting for year of first exposure, and restricting follow-up to 1978 onwards-and their potential effects on the results. These were touched on in the paper, but perhaps not to the detail that was warranted. Farioli et al (2014) requested additional analysis, which has been completed and is presented here.

Table 1 shows the results of a multivariable generalised gamma accelerated failure-time model that includes only cases first exposed to asbestos between 1950 and 1969 to limit left and right censoring, and analysing the entire followup period (1971-2005) rather than restricting it to post 1978. In addition, results are also presented for the same multivariable model but avoiding adjustment for the year of first exposure, as requested by Farioli et al (2014). Unfortunately, it was not possible to undertake the stratum-specific analysis as suggested by Farioli et al (2014), owing to the relatively small number of cases in each stratum.

Only including cases exposed for the first time between 1950 and 1969, and analysing the entire follow-up period (1971-2005) did not greatly influence the results in comparison to those presented in the original paper (Table 1). However, avoiding adjustment for the year of first exposure resulted in three notable differences: the latency period for women was no longer statistically significantly longer than that for men; asbestos removal workers now observed a statistically significantly shorter latency period than insulation workers; and there was now a statistically significant association between duration of exposure and latency, with latency tending to increase with duration (Table 1).

It is well known, and intuitive, that estimates of latency are related to the duration of follow-up. If a cohort has been followed up for just 20 years since their first exposure to asbestos, then only shorter mesothelioma latencies of 20 years or less would be possible within the follow-up period and so any estimate of median latency would be an underestimate (given the long latency for mesothelioma). Peto highlighted this, and the relationship between latency and both age at exposure and mortality due to other causes, in his 1985 paper (Peto, 1985). It was therefore expected that there would be a strong association between latency and the year of first exposure, with latency decreasing as the year of first exposure increases. In fact, this was one of the main reasons for including this variable in the final multivariable model that was presented in the paper.

Farioli et al (2014) commented that the time since first exposure to asbestos should not be adjusted for because of its spurious association with latency. However, I propose that this is precisely why it should be included. For example, asbestos removal work only started to become prominent in Great Britain in the 1980s with asbestos insulation work being much earlier, and so this group will tend to have shorter follow-up times and hence a spuriously shorter average latency than they would if they had the same follow-up time as insulation workers. Therefore, without attempting to adjust for differences in follow-up through variables such as time since first asbestos exposure, the true effect of occupation (if any) could be distorted. However, this is not an issue in the analysis presented in Table 1, which restricts cases to only those that were first exposed between 1950 and 1969.

Not adjusting for time since first asbestos exposure results in a trend of increasing latency with increasing duration of exposure (Table 1). As touched upon in the original paper and in the comment by Farioli et al (2014), this is not unexpected given what we know about latency-for example, in order to have experienced $>30$ years of exposure to asbestos, an individual could not have died with mesothelioma within 30 years of their first exposure to asbestos. Latency, year of first exposure and duration of exposure are all closely related, and so adjusting for time since first exposure removed this spurious association between duration and latency (Table 1).

Finally, Farioli et al (2014) commented on the choice to restrict follow-up to when information on asbestosis was available (from 1978 onwards), rather than including the full follow-up period. This choice was made because having asbestosis is an important indicator of the intensity of exposure to asbestos and so was of interest in its own right, rather than being included purely to adjust for potential confounding. A sensitivity analysis conducted at the time, and now the results presented here, confirmed that including all follow-up rather than restricting this to 1978 onwards made little difference to the results. Hence I presented the results using the restricted follow-up and including death with asbestosis, with an analysis of the full follow-up time serving as a sensitivity analysis.

There were three main indicators of intensity of asbestos exposure specified in the original paper that were used to judge the strength of support for the intensity hypothesis: sex, presence of asbestosis and occupation. The additional analysis presented here did not allow presence of asbestosis to be included, and so the judgement here relies on sex and occupation. The difference in mesothelioma latency with sex was in the direction expected if the intensity hypothesis was true, but it was not statistically significant when not adjusted for year of first exposure. In addition, the difference in latency between insulation workers and removal workers was in the opposite direction to that expected if the hypothesis was true. Hence my conclusion from the original paper remains unchanged; this study found no evidence that greater intensity asbestos exposure would lead to shorter mesothelioma latencies.

I would also like to take this opportunity to remark on the comment mentioned by Farioli et al (2014) and made by Consonni et al (2014) and Mirabelli and Zugna (2014), that the analysis should have included all individuals in the cohort and not just those who died with mesothelioma. This is a point that was considered before undertaking the analysis, but a number of problems arise if all individuals are included. First, $<1 \%$ of individuals in the cohort died from mesothelioma during follow-up. Therefore, if individuals who died from other causes or were alive at the end of follow-up were treated as censored observations, then the median latency would not be estimable using classical methods. In addition, any median latency predicted from survival analysis would be longer than the life expectancy of individuals in the cohort-the predicted median latency from an empty generalised gamma accelerated failure-time model using data from the full cohort was 115 years. 